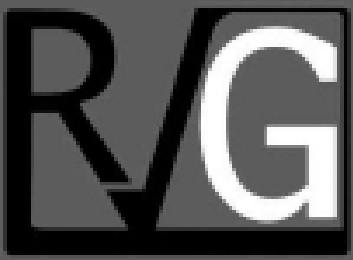

Año 24 No. 88

Octubre - Diciembre 2019

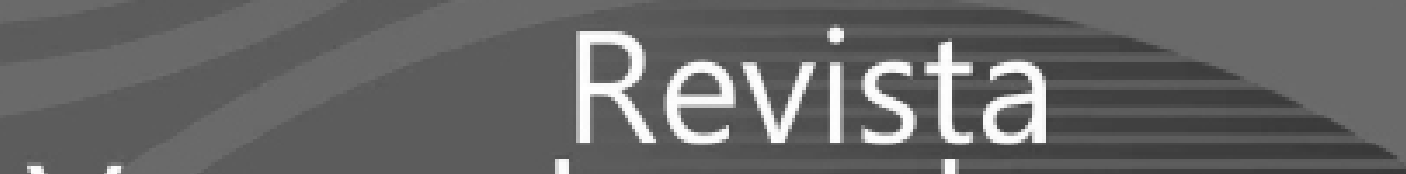

Venezolana de

verencla

UNIVERSIDAD DEL ZULIA (LUZ)

Facultad de Ciencias Económicas y Sociales

Centro de Estudios de la Empresa 


\title{
Perspectiva teórica del diagnóstico organizacional
}

\author{
Bravo Rojas, Leonidas Manuel ${ }^{1}$ \\ Valenzuela Muñoz, Alberto ${ }^{2}$ \\ Ramos Vera, Patricia María ${ }^{3}$ \\ Tejada Arana, Arístides Alfonso ${ }^{4}$
}

\section{Resumen}

El diagnóstico organizacional es un estudio especializado que requiere toda organización y se encarga básicamente de evaluar la situación estratégica actual de la empresa con una visión a futuro, por tal motivo, el objetivo de este trabajo se centra en el estudio del diagnóstico organizacional, sus componentes e importancia, para lo cual se llevó a cabo una investigación documental descriptiva a través de la de recolección de datos de fuentes bibliográficas, usando como referentes las propuestas de Francés

\section{Recibido: 20-07-19 Aceptado: 15-09-19}

1 Doctor en Administración, Magíster en Administración de negocios y relaciones internacionales MBA, Ingeniero industrial, Coordinador de investigación. Escuela Profesional Ingeniería Industrial Campus Lima Norte Universidad César Vallejo Escuela Profesional de Ingeniería Industrial, Perú. Email: Ibravo@ucv.edu.pe ORCID: 0000-0001-7219-4076

2 Doctor en economía. Especialista en finanzas públicas, Proyectos de inversión pública, costos, finanzas empresariales, Evaluador de proyectos de inversión, Cargos desempeñados como Vicepresidente administrativo en UNIA, Jefe de planificación y presupuesto en UNJFSC, Director de la escuela de economía y finanzas en UNJFSC, jefe de departamento académico en UNJFSC, docente de post grado en Universidad San Pedro, Universidad Inca Garcilaso de la Vega y UNJFSC, investigaciones realizadas en ciencias sociales y agropecuarias, actualmente se desempeña como Vicepresidente de investigación en Universidad Nacional Autónoma de Tayacaja Daniel Hernández Morillo (UNAT), Perú. Email: albertovm50@hotmail.com ORCID:0000-0002-2272-5307

3 Magister en Educación con Mención en Docencia y Gestión Educativa, Licenciado en educación secundaria especialidad matemática, Segunda Especialidad en Investigación y Didáctica en el área de matemática, Nivel de educación secundaria de Educación Básica Regular. Docente de la Universidad Norbert Wiener y Doctorando en Educación Universidad Privada César Vallejo, Perú. Email: patriciadaro@gmail.com ORCID: 0000-0002-7591-964X

4 Doctor en Administración y Economía, Máster universitario en Gestión y Comunicación de Entidades Sociales y Solidarias, Maestro en Ciencias Económicas e Ingeniero Administrativo. Docente de la Escuela de pos grado Universidad César Vallejo. Perú. Email: atexada@gmail.com ORCID: 0000-0002-8905-3082 
(2001), Thompson y Strickland (2004), David (2003), Peters y Norhaus (2005), Schroeder (1992), Porter (2000). Entre los aspectos que destacan estos autores resalta que el diagnóstico organizacional presenta varias elementos que actúan de manera sistémica y organizada, factores internos y externos que se conjugan para constituir estrategias de adaptación y sobrevivencia para la organización, teniendo como conclusión que manejado de manera adecuada le permite a las organizaciones prever y planificar de manera oportuna cualquier incertidumbre o amenaza a la cual se vea sometida, asimismo, necesita de otros elementos y de la integración de recursos y capital humano para garantizar su éxito.

Palabras clave: diagnóstico organizacional; factores internos; factores externos; organización; planificación.

\title{
Theoretical perspective of organizational diagnosis
}

\begin{abstract}
The organizational diagnosis is a specialized study that requires any organization and basically is responsible for evaluating the current strategic situation of the company with a vision for the future, for this reason, the objective of this work is focused on the study of the organizational diagnosis, its components and importance, for which a descriptive documentary investigation was carried out through the collection of data from bibliographic sources, using as reference the proposals of French (2001), Thompson and Strickland (2004), David (2003), Peters and Norhaus (2005), Schroeder (1992), Porter (2000). Among the aspects highlighted by these authors, it is worth highlighting that the organizational diagnosis presents several elements that act in a systemic and organized way, internal and external factors that combine to constitute adaptation and survival strategies for the organization, having as a conclusion that properly managed It allows organizations to foresee and plan in a timely manner any uncertainty or threat to which it is subjected. It also needs other elements and the integration of resources and human capital to guarantee its success.
\end{abstract}

Keywords: organizational diagnosis; internal factors; external factors; organization; planning

\section{Introducción}

El mundo empresarial de hoy en día está no solo en la búsqueda de la mejora de sus procesos sino en la renovación, incluso, de sus planes de negocios para garantizar rentabilidad en el plazo planeado y permanencia en el mercado. Todas las decisiones importantes son tomadas en los procesos de planeación estratégica, por lo que se requiere de una visión sistemática de la empresa y 
su entorno en favor de la competitividad y productividad.

En este sentido, el diagnóstico organizacional es un estudio especializado que requiere toda organización y se encarga básicamente de evaluar la situación estratégica actual de la empresa, sus debilidades reflejadas en sus problemas; así como, sus potencialidades y su alcance, incluyendo su crecimiento. La intención principal de un diagnóstico organizacional es de proveer al interesado una visión panorámica de la empresa en búsqueda de generar eficiencia a través de cambios sustanciales. Se dice que las organizaciones, con todas sus implicaciones, forman parte del resultado de la búsqueda que ha encauzado el hombre racional a la prosperidad (Guísar, 2013).

Un diagnóstico organizacional elaborado sistemáticamente, abordando todo sus componentes, se convierte en estrategia fundamental para el logro de los objetivos empresariales, evitando improvisaciones y asegurando la rentabilidad, posicionamiento y competitividad de la empresa, por lo estratégico de la aplicación de un diagnóstico organizacional, es que se desarrolla este trabajo que tuvo como objetivo hacer un recorrido teórico por el diagnóstico organizacional, sus componentes e importancia, para lo cual se empleó una investigación de tipo documental-a través de la revisión de fuentes bibliográficas diversas.

\section{Diagnóstico organizacional: una visión desde la teoría}

Para comprender que implica el diagnóstico organizacional se hace necesario inicar con su definición, desde la perspectiva de diferentes autores, en este sentido, para Francés (2001), el diagnóstico organizacional consiste en el análisis del entorno para identificar las oportunidades y amenazas de la empresa, así como, el análisis interno para identificar las fortalezas y debilidades. En tanto, Thompson y Strickland (2004), sugieren que el diagnóstico implica considerar dos grupos de factores: 1) las condiciones competitivas y de la industria y 2) las capacidades competitivas, recursos, fortalezas y debilidades internas, y la posición en el mercado que ocupan las organizaciones.

Un análisis integral de la situación de cualquier empresa, orientado a generar un diagnóstico veraz y oportuno, abarca un proceso complejo, constituido por factores externos e internos que afectan la toma de decisiones de dicha organización. En este orden de ideas, David (2003), plantea la necesidad de una auditoría integral de la organización, para abarcar la evaluación del entorno (factores externos) y la evaluación de las fortalezas y debilidades de la empresa (factores internos).

Con base alo anterior, el diagnóstico organizacional implica el análisis de los aspectos externos y su impacto en la organización, equiparándolos, a su vez, con los aspectos internos, la evaluación de estos escenarios permite diseñar estrategias de maximización de oportunidades y de minimización de amenazas haciendo uso eficiente de los recursos organizacionales.

En otro orden de ideas, con el fin de lograr dilucidar lo referente a la situación estratégica de la empresa, es necesario el estudio de las políticas, normas, funciones y acciones organizacionales como centro de actividad en el proceso de planeación el cual está dirigido directamente por los gerentes de la 
compañía, en los diferentes niveles organizacionales, alta gerencia, media o baja, siendo las planificaciones estratégicas, operativas o funcionales la directriz para la ejecución y toma de decisiones.

Vale resaltar, el origen de la definición de estrategia, este término se originó en el campo militar y se puede afirmar que el libro más antiguo e influyente que explica su definición y aplicación en el área gerencial es el arte de la guerra de Tzu (1963) el cual fue escrito aproximadamente 500 años A.C. El significado etimológico de estrategia proviene de la palabra griega strategos que significa líder de ejército esquema que ha sido implementado por las organizaciones militares para intentar alcanzar sus objetivos.

Al respecto, Tzu (1963) afirma que el arte de la guerra considerada como la estrategia es de vital importancia para el Estado, convirtiéndose en un factor de vida o muerte, para el caso gerencial se trata de la supervivencia de la empresa. La estrategia sería entonces un camino que conduce al éxito, en caso de ganar ventaja o a la ruina en caso de perderla. Así mismo, hace mención a cinco factores: el camino como guía que induce al pueblo a tener el mismo objetivo que sus dirigentes; el clima; el terreno en términos de distancia, facilidad o dificultad de desplazamiento y seguridad; la autoridad, como el valor de la inteligencia, honradez y severidad; y por último, la disciplina en la organización, cadena de mando y logística. En general el que tenga claro estos factores alcanzará la victoria (Tzu, 1963).

Por su parte, Francés (2006), define la estrategia como la combinación de medios que se emplean para alcanzar los objetivos en presencia de incertidumbre, de hecho cuando se tiene certeza de la eficacia de los medios no se requiere la formulación estratégica. El autor, plantea el asunto de la planificación estratégica como un proceso en el cual se logran definir de forma sistemática los lineamientos estratégicos o líneas maestras de la empresa u organización, los cuales se desarrollan como guías a detalle para la acción, se asignan los recursos y se plasman por escritos como planes. Adicionalmente, la planificación estratégica toma en cuenta la incertidumbre a través de la identificación de oportunidades y amenazas en el entorno y anticipa a su vez lo que los actores pueden hacer.

Ampliando la definición de planeamiento estratégico, está compuesta por la visión, misión y los objetivos clave (Carrión, 2003). La visión se refiere a lo que se quiere llegar y es una declaración de las intenciones de la situación que se pretende alcanzar en el futuro, lo cual debe ir más allá de los objetivos financieros y debe involucrar al personal. Por otro lado, la misión responde a la interrogante de la esencia de lo qué hace la empresa mostrando su razón de ser, siendo más específica que la visión de la empresa.

Un análisis estratégico se desarrolla, por lo tanto, a través de tres etapas, según Gimbert (2003), la primera se denomina como planeación estratégica corporativa que involucra la misión, la visión y los objetivos, ya previamente mencionados; la segunda etapa, se trata de la planeación estratégica funcional donde se ejecutan los proyectos estratégicos basados en lo establecido en la primera etapa, la tercera parte es la planeación estratégica operativa en donde se ponen en marcha los planes de acción de la organización.

Por último, para ejecutar los planes 
es necesario llevar a cabo un análisis estratégico interno y externo en las organizaciones mencionando desde el inicio del desarrollo organizacional y el cual puede ser estructurado desde el modelo de las 5 fuerzas de Porter (1999), uno de los modelos más frecuentemente utilizados en el campo de la gerencia, más adelante se hará una descripción del mismo.

Vale aclarar que este no es el único modelo disponible para realizar análisis estratégicos organizacionales, entre los más comunes también se encuentra el análisis FODA, el cual considera tanto los factores del entorno como los internos, para evaluar a las organizaciones, generalmente, este análisis situacional sirve como base para el desarrollo de otros.

Por otro lado, para el análisis de los factores exógenos que condicionan el desempeño de la organización, tanto sus aspectos positivos (oportunidades) como negativos (amenazas), también resultan de utilidad las matrices de evaluación de los elementos tanto externos como internos, entre los que resaltan: la Matriz de Evaluación de Factores Externos (MEFE) y la Matriz de Evaluación de Factores Internos (MEFI).

El uso de estas matrices permite inferir, luego de un proceso que implica análisis, discusión y evaluación, oportunidades y amenazas que viabilizan o impiden el logro de los objetivos establecidos y la misión y visión del negocio. Resaltan entre los factores externos: los económicos, políticos, legales, gubernamentales, sociales, culturales, demográficos, ambientales, tecnológicos, competitivos. También permiten la identificación de fortalezas y debilidades a nivel interno de la organización.

A continuación, se presentan los planteamientos teóricos de autores tales como Thompson y Strickland (2004), David (2003), Hitt et al, (2004), Francés (2001), Porter (2000) expertos en materia de diagnóstico, con la finalidad de exponer los aspectos más importantes que se deben considerar en esta temática.

\subsection{Factores externos}

Para Thompson y Strickland (2004), los factores externos están constituidos por todas las fuerzas que están fuera de los límites de la compañía; considerándose relevantes porque son lo suficientemente importantes como para afectar la toma de decisiones dentro de la entidad, por su parte, David (2003), define las fuerzas externas como las situaciones que se traducen en cambios en la demanda de los consumidores, lo cual afecta los tipos de bienes y servicios desarrollados, el posicionamiento estratégico empresarial, las estrategias de segmentación del mercado y la selección de empresas para adquirir o vender.

En función de lo expuesto anteriormente, se hace evidente que los factores externos representan variables que quedan fuera del dominio de las organizaciones; no obstante, afectan la gestión interna, razón por la cual deben ser monitoreadas y vigiladas para adaptar las estrategias organizacionales a los cambios del entorno, ya sea por un impacto negativo o, por el contrario, por beneficios que los factores externos puedan presentar.

Según, Hitt et al, (2004), entre los factores más importantes que afectan la gestión de las organizaciones están los factores económicos, referidos al carácter y al curso de la economía en el lugar donde ésta compite o podría 
hacerlo, considerando, además, el monitoreo, pronóstico y evaluación de las economías extranjeras debido a la interrelación de mercados causada por el fenómeno de la globalización, de esta forma se busca detectar posibles amenazas y oportunidades.

Puntualmente, Francés (2001), comparte los planteamientos de David (2003), al identificar como variables clave para la evaluación del contexto económico de las empresas, el comportamiento de los siguientes elementos: política monetaria, política fiscal, cambios en la demanda, fluctuaciones de precios, disponibilidad de créditos, patrones de consumo, tasas de intereses, acuerdos de integración, inflación, entre otros factores, que afectan la gestión en las organizaciones. Las organizaciones constantemente sufren las consecuencias o disfrutan los beneficios derivados de los factores económicos mencionados anteriormente.

Asimismo, David (2003), hace mención a otros factores importantes para el diagnóstico de las organizaciones, a los cuales denomina factores sociales; estos factores ejercen un impacto determinante en el comportamiento de todos los bienes, servicios, mercados y clientes, es decir, definen la forma de vida, de trabajo, de producción y de consumo de la población, llamando la atención de todas las organizaciones, sean lucrativas o no lucrativas. Al respecto, Thompson y Strickland (2004), plantean que los factores sociales pueden constituirse en fuerte promotores de cambios en la demanda de la industria.

Según, Hitt et al, (2004), los factores socio-culturales se refieren a los valores culturales de una sociedad, debido a que éstos representan la base de toda organización social, y generalmente impulsan los cambios demográficos, económicos, tecnológicos y políticos. En cuanto a los cambios demográficos, el autor plantea la intervención de diversas variables entre las cuales se encuentran: tamaño de la población, estructura de los grupos de edad, distribución geográfica, composición étnica y distribución del ingreso.

Otro factor considerado por David (2003), es el ambiental, referido a las características físicas y climáticas, y las consecuencias de las operaciones empresariales en la degradación del medio ambiente, como una de las mayores amenazas para la sociedad. Para Sapag y Sapag (2008) toda organización debe gestionar el diseño de un proceso productivo orientado a considerar el mejoramiento ambiental en toda su cadena de transformación.

Además de los factores ambientales descritos anteriormente, toda organización se encuentra inmersa en un ambiente político y jurídico, el cual, según Hitt et al (2004), compiten por influir en el rumbo de las políticas gubernamentales y en la finalidad de las leyes que rigen las actividades económicas de cualquier país. Fisher y Espejo (2004) mencionan que los factores políticos y legales consisten en un conjunto interactuante de leyes, agencias gubernamentales y grupos de presión que influyen y limitan la conducta de organizaciones y personas en la sociedad.

David (2003) y Thompson y Strickland (2004), plantean que las acciones de regulación, implementadas por los gobiernos locales, generan cambios importantes en las prácticas gerenciales y en lo enfoques estratégicos desarrollados por las, representando oportunidades o amenazas clave para las mismas. En la actualidad, es de 
carácter estratégico, para cualquier organización, analizar la filosofía de gestión que tienen los gobiernos locales en donde desarrollan sus operaciones. Las acciones gubernamentales generalmente abarcan: promulgación de leyes referidas al sector de la empresa, ordenamientos fiscales, políticas de regulación, leyes relacionadas con los trabajadores, financiamiento de instituciones educativas, entre otros.

Para complementar lo expuesto, Thompson y Strickland (2004), consideran para el diagnóstico organizacional a los factores tecnológicos como un elemento importante, es decir, según los autores, éstos tienen el poder de influir determinantemente en el contexto de cualquier sector económico, a través del desarrollo de nuevos productos que pueden salir al mercado a un menor costo y con la posibilidad de abrir las fronteras del sector ante los consumidores.

El desarrollo de tecnología aplicada a cualquier producto de, puede originar un efecto directo sobre el nicho de mercado. Productos avanzados logran ampliar la base de clientes de una empresa, revitaliza el crecimiento y amplia el grado de diferenciación de sus productos. Según David (2003), los avances tecnológicos tienen el poder de revolucionar cualquier industria y un ejemplo de ello es el Internet, que funge como un motor global de los negocios, esto permite el estímulo de la productividad de los países y sirve como medio para mejorar la calidad de vida de sus habitantes.

Adicional a los factores anteriores, existen factores como los competitivos, los cuales según Francés (2001), representan otro elemento estratégico a considerar en el diagnóstico organizacional externo, debido a que según el autor, en los últimos años se ha asumido la idea de la competencia en el mercado, la cual no se desarrolla al considerar sólo las condiciones de cada organización, es decir, se expande hasta el contexto global de los sectores industriales pertenecientes a una economía.

El éxito de una planificación estratégica, para cualquier organización, depende en gran medida del análisis y diagnóstico del sector industrial al cual ésta pertenece (David, 2003), para el autor la recolección y evaluación de la información sobre los competidores del sector es un factor básico para la formulación de estrategias con éxito.

Además de lo anterior, existen otros modelos para realizar diagnósticos organizacionales, donde se asume un enfoque hacia la competitividad de los sectores industriales, tal es el caso del modelo planteado por Porter (1999), donde se expone el análisis de la competencia de un sector dentro de un determinado país. Dicho modelo parte del supuesto de que los participantes compiten entre sí para apropiarse de la mayor porción de los beneficios derivados del sector. de mercado

Para Porter (1999), los beneficios derivados de un sector económico dependen de cinco fuerzas: 1) la rivalidad entre empresa competidoras, 2) entrada potencial de nuevos competidores, 3) desarrollo potencial de productos sustitutos, 4) poder de negociación de los proveedores y 5) poder de negociación de los consumidores, las cuales se describen a continuación.

- Rivalidad entre empresas competidoras: es considerada la fuerza más poderosa de las cinco presentes. Plantea que el éxito de una organización, consiste en lograr el alcance de una ventaja 
competitiva en sus estrategias sobre el resto de las empresas que conforman el sector.

- Entrada potencial de nuevos competidores: esta fuerza plantea la estrategia de identificar las empresas nuevas con posibilidad de ingresar al mercado, con la finalidad de vigilar sus estrategias rivales, contraatacar y obtener beneficios de las fortalezas y oportunidades existentes.

- Desarrollo potencial de productos sustitutos: esta fuerza la representan las presiones derivadas de los productos sustitutos de empresas de otros sectores. La presión se origina porque se establece un tope en el precio de los productos ofrecidos, antes de que los consumidores empiecen a optar por el producto sustituto.

- Poder de negociación de los proveedores: esta fuerza plantea que las organizaciones deben tener una estrategia de negociación hacia atrás, para obtener el control de los proveedores.

- Poder de negociación de los consumidores: se refiere al poder de los consumidores en las negociaciones de compra y venta. Se plantea ofrecer garantías y servicios especiales para obtener un mayor poder en la negociación con los consumidores sobre el resto de la competencia. as cinco fuerzas propuestas por Porter (1999) tienen un mayor impacto en sectores económicos, donde la competencia entre las organizaciones que los conforman, está caracterizada por una intervención disminuida del Estado y atienden a las condiciones de oferta y demanda en un ambiente de libre mercado. La información antes presentada, se constituye en la base fundamental, para la realización del diagnóstico situacional externo en el sector objeto de estudio.

\subsection{Factores internos}

A lo interno, las organizaciones deben realizar igualmente un diagnóstico de sus recursos, partiendo de la premisa de que toda empresa posee fortalezas y debilidades en sus áreas funcionales es esencial la definición clara de estos elementos, ya que los mismos están bajo el control de la gerencia y, como tal, deben ser manejados de manera adecuada (David, 2003).

Considerando que, el análisis de los factores internos permitirá determinar las fortalezas y las debilidades de la empresa, respecto a sus competidores $y$, a partir de ellos, evaluar su capacidad para aprovechar las oportunidades y contrarrestar las amenazas, entonces, el conocimiento y dominio de las fortalezas de la organización demostrarán su superioridad frente a los competidores ya que se establecerán como ventajas competitivas

David (2003), plantea realizar dicho diagnóstico a través del análisis de factores internos que constituyen la organización tales como: gerencia estratégica, mercadotecnia, finanzas, producción y el manejo de los sistemas de información de la gerencia, los cuales se describen a continuación.

\section{- La gerencia}

Constituye el eslabón responsable de gestionar la eficiencia de factores internos de las organizaciones tales como: planeación, organización, motivación, recurso humano y control, 
con la finalidad de definir las estrategias necesarias para alcanzar el éxito en cada uno de ellos.

Para Hitt et al (2004), la gestión del recurso humano es fundamental, ya que abarca factores como: conocimientos de la organización, la confianza del personal y la capacidad de los administradores. Otro elemento a considerar en la gerencia, es su capacidad para fomentar la organización interna, considerada como la estructura de relaciones entre las tareas y la autoridad dentro de la empresa. (David, 2003)

\section{- Mercadotecnia}

Representa una herramienta que tiene como finalidad definir, anticipar, crear y satisfacer las necesidades de los clientes en cuanto a bienes y servicios. Para David (2003), existen siete (7) funciones básicas de mercadotecnia: a) análisis del cliente, b) venta de productos y servicios, c) planeación de productos y servicios, d) establecimientos de precios, e) distribución, f) investigación de mercados y g) análisis de oportunidades.

\section{- Factores financieros}

Frecuentemente se consideran como la posición competitiva de la entidad y la atracción más poderosa para los inversionistas. Las debilidades y fortalezas financieras determinan en muchas ocasiones el rumbo de las estrategias de una organización, que se diseñan al considerar factores financieros como: a) liquidez, b) financiamiento, c) capital de trabajo, d) rentabilidad, e) utilización de activos y f) flujo de capital (David, 2003).

El autor añade, que la gerencia de operaciones es la instancia de la organización encargada de gestionar la transformación de entradas al proceso, tales como: materia prima, mano de obra, capital, maquinaria e instalaciones, en productos terminados. La gerencia de operaciones y producción según Schroeder (1992), comprende cinco áreas básicas de decisión: a) proceso, b) capacidad, c) inventario, d) fuerza laboral y e) calidad

\section{- Sistemas de información}

Generan la información que constituye la piedra angular de todas las organizaciones pues proporcionan la base para la toma de decisiones gerenciales. Por esta razón, el manejo oportuno de información puede potenciar las fortalezas y disminuir las debilidades internas en una empresa. El sistema de información es la herramienta de que dispone una organización para recaudar, almacenar, sintetizar y presentar la información veraz y pertinente para la toma de decisiones (David 2003).

En este mismo orden de ideas, existen otros factores internos de interés que ejercen influencia en la gestión de una empresa y vale la pena mencionar, estos son:

\section{- Estructura organizativa}

La estructura organizacional es aquel conjunto de relaciones estables entre los roles de una empresa, ésta define cómo se dividen, agrupan y coordinan las tareas de una organización; la estructura organizativa debe ir alineada a las estrategias establecidas; sin embargo, la estructura establece límites a la definición de las estrategias, es decir, la organización no puede formular éstas sin tomar en cuenta los recursos de los cuales dispone.

En función de la estructura 
establecida se definen procesos de trabajo, los cargos y las responsabilidades asociados a los mismos y se evidencian las relaciones jerárquicas y responsabilidades de las áreas funcionales de la organización. Una adecuada estructura conlleva a la eficiencia en el trabajo (Francés, 2001).

\section{- Planeación y Control}

El cambio es lo único constante en una organización y la planeación, entendida como el puente entre el presente y el futuro de una organización, debe asumir los cambios existentes en el entorno. La planeación no es más que el proceso por medio del cual se determina si se debe realizar una actividad o tarea, se constituye en un mecanismo eficaz para alcanzar los objetivos propuestos $y$, ayuda a la empresa a obtener el efecto máximo de un esfuerzo dado, de igual forma permite reunir los recursos necesarios y realizar las tareas de la manera más eficiente posible, con miras a evitar el menor desperdicio y obtener una utilidad justa (David, 2003).

La planeación ejerce un impacto positivo en el rendimiento de la empresa y de su recurso humano, pues permite identificar y aprovechar las oportunidades externas, y al mismo tiempo minimiza el efecto de la amenaza. La planeación ofrece a una empresa la posibilidad de adaptarse a mercados cambiantes y poder trazar su rumbo desarrollándose como una organización proactiva más que reactiva.

La planeación se realiza a nivel de toda la organización, es decir, considera un enfoque global de la empresa, define planes estratégicos sobre los cuales se establecen objetivos y estrategias generales que conducen a una gran variedad de actividades. Sobre la base de la planeación estratégica es donde se elaboran los demás planes de la empresa, tanto los tácticos como los operativos, razón por la cual un plan estratégico no se puede considerar como la suma de éstos, sino, como uno que los contiene a todos (David, 2003).

Como todo planeamiento, la planeación estratégica es móvil y flexible, cada cierto tiempo se debe analizar y hacer los cambios necesarios. Asimismo, es un proceso interactivo que involucra a todos los miembros de la empresa, los cuales deben estar comprometidos con ella y motivados al logro de los objetivos.

En la realización de las actividades planeadas debe estar garantizada la función de control que permite la conducción de estas, es decir, es necesario el control para medir el rendimiento y detectar a tiempo las deviaciones, de esta manera, es posible aplicar las acciones correctivas y reducir al mínimo los efectos negativos. De acuerdo con David (2003) el control en la realización de todas las actividades se basa en cuatro pasos básicos.

- El establecimiento de normas de rendimiento.

- La medición del rendimiento individual y de la empresa.

- La comparación del rendimiento real con las normas de rendimiento planeadas.

\section{- La toma de acciones correctivas.}

Govindarajan y Anthony (2003), plantean que el control de la gestión se encuentra entre la formulación de la estrategia y el control de las operaciones, es decir, la formulación está enfocada a largo plazo y el control se centra en las actividades a corto plazo, la formulación 
de las estrategias nace de datos inciertos del futuro, por su parte el control de las actividades utiliza datos reales y exactos, entonces el control de la gestión es un punto intermedio entre los datos futuros y los datos reales del momento.

\section{- Objetivos y Estrategias}

En toda organización se definen objetivos, como guías de acción para lograr el fin establecido y estipulado en los planes elaborados, estos objetivos se desagregan por áreas funcionales y se definen para su cumplimiento estrategias para lograrlos. Francés (2001) plantea que todos los seres humanos tienen la capacidad de fijarse objetivos y dirigir las acciones para alcanzarlos, correspondientemente, se habla de las estrategias y tácticas necesarias para su consecución, las cuales se definen según Thompson y Strickland (2004) como las acciones y enfoques de negocio empleados por la administración para lograr un adecuado desempeño organizacional.

En las organizaciones, la estructura definirá el funcionamiento de su gestión, establecerá las pautas para la definición de objetivos y éstos a la vez se constituirán en la base para la definición de estrategias, en función de lograr lo estipulado en los planes estratégicos, tácticos y operacionales establecidos en los diferentes niveles de la estructura organizativa de la empresa.

Otra herramienta de diagnóstico es la desarrollada por Peters y Waterman (1990) basada en un modelo que une los 7 factores básicos para cualquier estructura organizativa, denominado Las 75 de McKinsey (figura 1), este enfoque suele emplearse para evaluar si la implementación de las estrategias en una organización está alineada con dichos factores. En caso negativo sería necesario replantearse parte o la totalidad de la estrategia. Estos factores son:

1. Style (estilo): el estilo es la cultura de la organización. Normalmente es la cúpula quien debe establecer las bases de los comportamientos y buenas prácticas que marcarán el estilo y la forma de ser de la empresa. Además, deben ser los directivos y jefes los primeros en dar ejemplo al resto de empleados de la empresa.

2. Staff(recurso humano): los empleados son la columna vertebral de cualquier organización y uno de sus más importantes activos. Es por ello que la forma de tratar a los recursos humanos debe estar alineada con la estrategia.

3. Systems (sistemas): incluye los procesos internos y los sistemas de información que posibilitan el funcionamiento de la empresa. Los procesos y la información pueden compararse con la sangre al fluir por un cuerpo.

4. Strategy (estrategia): se basa en la manera de organizar y enfocar los recursos, para conseguir los objetivos de la organización. Podría compararse con el cerebro de una organización.

5. Structure (estructura): es la manera mediante la cual se organizan, se relacionan e interactúan las distintas variables y unidades del negocio. La estructura puede ser departamental o no, con una jerarquía lineal, matricial, divisional o de otro tipo. Asimismo, se puede dividir geográficamente (local, estatal o plurinacional), de gestión centralizada o descentralizada. 
También la estructura puede depender de la fórmula jurídica que tiene la entidad (sociedad anónima, limitada, cooperativa) y el modelo de expansión buscada (franquicias, orgánica, fusiones).

6. Skills (habilidades): se refiere a las habilidades y capacidades requeridas por los miembros de la organización. Es lo que Porter llama Competencias Centrales. También puede referirse al knowhow de la compañía.

7. Sharedvalues(valorescompartidos): los valores compartidos son el corazón de la empresa. Lo que une a sus miembros y alinea a todos ellos en la misma dirección.

Los elementos anteriores pueden orientar la realización del diagnóstico interno en las organizaciones, en este sentido, ambos grupos de factores, tanto los externos como los internos, representan información básica para la definición de estrategias y la toma de decisiones en entornos altamente cambiantes y volátiles, como los que actualmente atraviesa Venezuela y dentro de este sus estados y las empresas que conforman el sector empresarial.

Adicionalmente, la utilización de alguno de los modelos de orientación para el diagnóstico organizacional, permite ampliar la visión al momento de la comprensión integral de la cadena de suministro en un sector estratégico como pudiera ser el sector alimenticio gestionado, en muchos casos, apegado a concepciones tradicionales y altamente empíricas.

\section{Conclusiones}

El diagnóstico organizacional es un instrumento de gran valor para aquellas organizaciones que apuestan por el crecimiento y la competitividad, sobre el tema existen diferentes aportes teóricos traducidos en modelos que sirven para el análisis organizacional y, consecuentemente, para la definición de estrategias acordes a los resultados obtenidos.

sin lugar a dudas, el diagnóstico organizacional representa una pieza clave en el proceso de consultoría, asimismo, se constituye en herramienta básica para detectar cualquier amenaza hacia la organización y, a su vez, corregirla, principalmente, prepara a la empresa para aprovechar las oportunidades con base a las fortalezas favoreciendo la creación de estrategias que sirven como mecanismo de defensa ante cualquier situación.

En este sentido, un buen diagnóstico permite conocer el entorno organizacional, reduce la incertidumbre, apuntando a una mayor estabilidad y aumenta su competitividad en contextos altamente desafiantes y globales, aun cuando no resulta fácil, diagnosticar oportunamente la situación empresarial es una habilidad gerencial que debe ser potenciada y acompañada por un equipo de alto desempeño.

Es importante resaltar que el diagnóstico, como tal, es un paso previo pero sumamente importante para la definición de la situación actual de la empresa, posterior, se hace necesario el diseño de los planes y la ejecución de las acciones correspondientes, así como, la asignación de recursos para el logro de los objetivos planteados.

\section{Referencias bibliográficas}

David, Fred (2003), Conceptos de Administración Estratégica. Novena edición. Pearson Education. México. 
Fischer, Laura y Espejo, Jorge (2004), Mercadotecnia. Tercera Edición. Mc Graw Hill. 267 p.

Francés, Antonio (2001), Estrategia para la Empresa en América Latina. IESA. Caracas, Venezuela.

Francés, Antonio (2006), Estrategias y planes para la empresa con el cuadro de mando integral. Pearsons Educación de Mexico S.A

Gimbert, Xavier (2003), El enfoque estratégico de la empresa. Grupo planeta GBS.

Govindarajan, V. y Anthony, R (2003), Sistemas de control de gestión. Décima edición. McGraw Hill. España.

Hitt, Michael; Ireland, R. Duane y Hoskisson, Robert (2004), Administración Estratégica. Séptima edición. Cengage Learning Editores. México

Peters, Tom y Waterman, Robert (1990), En busca de la excelencia (Experiencia de las empresas mejor gerenciadas de los Estados Uni- dos). Reimpresión Editorial Norma. Colombia.

Porter, Michael (1999), Ser competitivo. Onceava edición. Editorial Deusto. España.

Porter, M. (2000), Estrategia competitiva: técnicas para analizar a las industrias y la competencia. $27 \mathrm{ma}$. Edición, Méjico. CECSA.

Samuelson, Paul y Nordhaus, William (2005), Economía. Decimoctava edición. McGraw Hill.

Sapag, Nassir y Sapag, Reinaldo (2008), Preparación y evaluación de proyectos. Quinta edición. McGraw Hill. México.

Thompson, Arthur y Strickland, A.J. (2004), Administración Estratégica. Decimotercera edición. McGraw Hill. México.

Tzu, S.; Lawson, J. (1963), El arte de la guerra para ejecutivos y directivos. Ediciones Obelisco, Barcelona. España.

- Esta obra está bajo una licencia de Creative Commons Reconocimiento-NoComercial- Compartirlgual 3.0 Unported. http://creativecommons.org/licenses/by-nc-sa/3.0/deed.es_ES 